\title{
Josephson junction analog and quasiparticle-pair current
}

\author{
Bak, Christen Kjeldahl; Pedersen, Niels Falsig
}

Published in:

Applied Physics Letters

Link to article, DOI:

$10.1063 / 1.1654590$

Publication date:

1973

Document Version

Publisher's PDF, also known as Version of record

Link back to DTU Orbit

Citation (APA):

Bak, C. K., \& Pedersen, N. F. (1973). Josephson junction analog and quasiparticle-pair current. Applied Physics Letters, 22(4), 149-150. https://doi.org/10.1063/1.1654590

\section{General rights}

Copyright and moral rights for the publications made accessible in the public portal are retained by the authors and/or other copyright owners and it is a condition of accessing publications that users recognise and abide by the legal requirements associated with these rights.

- Users may download and print one copy of any publication from the public portal for the purpose of private study or research.

- You may not further distribute the material or use it for any profit-making activity or commercial gain

- You may freely distribute the URL identifying the publication in the public portal

If you believe that this document breaches copyright please contact us providing details, and we will remove access to the work immediately and investigate your claim 


\title{
Josephson junction analog and quasiparticle-pair current
}

\author{
C.K. Bak and N.F. Pedersen
}

Physics Laboratory I, The Technical University of Denmark, DK-2800 Lyngby, Denmark

(Received 13 October 1972)

\begin{abstract}
A close analogy exists between a Josephson junction and a phase-locked loop. A new type of electrical analog based on this principle is presented. It is shown that the inclusion in this analog of a low-pass filter gives rise to a current of the same form as the Josephson quasiparticle-pair current. A simple picture of the quasiparticle-pair current, which gives the right dependences, is obtained by assuming a junction cutoff frequency to be at the energy gap.
\end{abstract}

It is well known ${ }^{1-3}$ that the total current density in a Josephson tunnel junction may be written as $^{3}$

$$
J=J_{1} \sin \phi+\sigma_{0}(V) V+\sigma_{1}(V)(\cos \phi) V
$$

where $d \phi / d t=2 e V / h, \phi$ being the phase, and $V$ the voltage across the junction. The first term in Eq. (1) represents the supercurrent, the second is the quasiparticle current, and the third is the quasiparticle-pair interference current. Recently there has been great interest in electrical ${ }^{4,5}$ and mechanical ${ }^{6}$ analogs that can simulate the Josephson junction. We present here a new scheme for an analog which is simple to build, and which can be operated at much higher frequencies than previously reported. A new and important feature of this analog is that it has inherent in it the $\cos \phi \operatorname{con-}$ ductivity of Eq. (1).

The analog is shown in Fig. 1 ; it is basically a phaselocked loop. The voltage-controlled oscillator (VCO) produces a voltage at an angular frequency $\omega_{0}+k V_{i}$, where $\omega_{0}$ is the carrier frequency, $V_{l}$ is the input voltage, and $k$ is a constant for the particular VCO. The output of the VCO and a signal at the carrier angular frequency $\omega_{0}$ are fed to the two inputs of a mixer. Using a low-pass filter with a cutoff angular frequency $\omega_{c}$, the voltage $V=V_{1} \sin \left(k V_{i} t\right)$ at the difference frequency is extracted.

If the effect of the filter at frequency $k V_{i}$ is neglected for the moment, the output of the current amplifier (transconductance $G$ ) is then

$$
I=I_{1} \sin \phi \text {, }
$$

where $I_{1}=G V_{1}$ and $d \phi / d t=k V_{i}$. Thus, the total current $I_{i}$ (Fig. 1) drawn by the loop input is

$$
I_{i}=I_{1} \sin \phi+V_{i} / R \text {. }
$$

For input current $I_{i}<I_{1}$, the phase of the VCO will lock to the carrier such that $I_{i}=I_{1} \sin \phi$. Equation (3) represents the usual Josephson element shunted by a quasiparticle resistance $R$. It will now be demonstrated that the inclusion of a filter transfer function causes a conductivity term similar to the $\cos \phi$ conductivity in Eq. (1).

To do this we will calculate the small-signal input admittance of the loop operating in the "zero-voltage" mode, i.e., the VCO phase locked to the carrier. An input current $I_{\mathrm{dc}}<I_{1}$ superimposed with a small rf current $I_{\mathrm{rt}}$ at angular frequency $\omega$ is applied, and by breaking the loop at point $P$ the loop gain $A=I / I_{i}$ is calculated. The termination at $P$ with a current generator $I_{\mathrm{dc}}$ as shown in Fig. 1 assures that the mean phase $\phi$ will remain unchanged. For small phase deviations $\mathrm{Eq}$. (2) may be rewritten

$$
I=I_{1} \sin \left(\phi+\int k V_{i} d t\right) \approx I_{1} \sin \phi+I_{1} \cos \phi \int k V_{1} d t,
$$

where $\sin \phi=I_{\mathrm{dc}} / I_{1}$. To calculate the loop gain at $\omega$, the ac part $I_{2 c}$ is taken to be

$$
I_{\mathrm{ac}}=I_{1} \cos \phi \int k V_{i} d t \text {, }
$$

which in the usual $j \omega$ formalism is

$$
I_{\mathrm{ac}}=I_{1}(\cos \phi) k R I_{\mathrm{rt}} / j \omega,
$$

where $V_{i}=R I_{\mathrm{rf}}$ has been inserted. By introducing a filter transfer function $\left(1+j \omega / \omega_{c}\right)^{-1}$, the loop gain at angular frequency $\omega$ is

$$
A=\omega_{1}(\cos \phi)(j \omega)^{-1}\left(1+j \omega / \omega_{c}\right)^{-1},
$$

where $\omega_{1}=k R I_{1}$. Separating real and imaginary parts yields

$$
A=-\frac{\omega_{1}}{\omega_{c}} \frac{\cos \phi}{1+\left(\omega / \omega_{c}\right)^{2}}-j \frac{\omega_{1}}{\omega} \frac{\cos \phi}{1+\left(\omega / \omega_{c}\right)^{2}} .
$$

The Nyquist diagram ${ }^{7}$ is illustrated in Fig. 2. From the shape of the curve we may conclude, according to Nyquist, ${ }^{7}$ that phase-locked loops containing not more than one upper cutoff frequency will be stable upon closing the loop. The closed-loop input admittance $Y$ $=Y^{\prime}(1+A)$, where $Y^{\prime}=1 / R$ is the open-loop input admittance. The effect of the filter is to push the gain curve away from the imaginary axis, which thus introduces a negative conductance component. The full expression for $Y$ is then

$$
Y=\frac{1}{j \omega} \frac{\omega_{1} \cos \phi}{R\left[1+\left(\omega / \omega_{c}\right)^{2}\right]}+\frac{1}{R}-\frac{1}{R} \frac{\omega_{1}}{\omega_{c}} \frac{\cos \phi}{1+\left(\omega / \omega_{c}\right)^{2}} .
$$

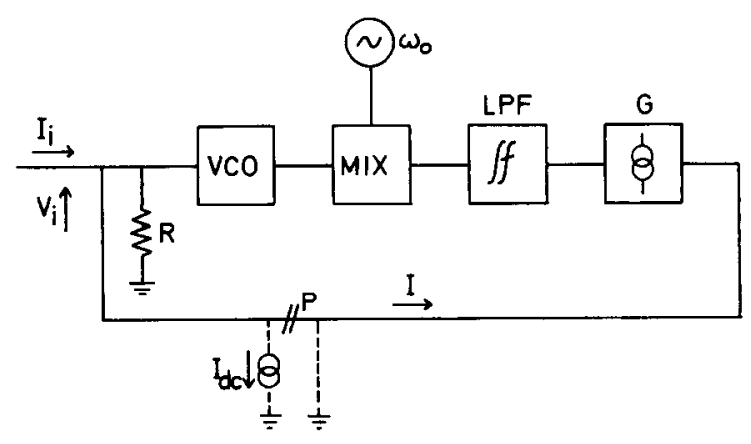

FIG. 1. Block diagram of the phase-locked loop. VCO, voltage controlled oscillator; MIX, mixer; LPF, low-pass filter; G, current generator. 


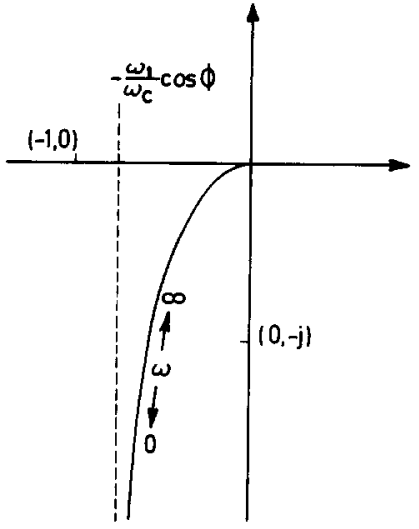

FIG. 2. Nyquist diagram. A plot of the open-loop gain $A$ in the complex plane with $\omega$ as a parameter.

In comparing Eq. (4) with the Josephson equation [Eq. (1)], the following things are readily apparent. The first term in Eq. (4) represents an inductance and can be identified with the supercurrent term in Eq. (1). The second term represents the quasiparticle conductivity, and the third term in Eq. (4) is identified as the quasiparticle-pair current in Eq. (1). The minus sign is important since it has been shown ${ }^{8,9}$ that $\sigma_{1}$ is actually negative.

At this point it is tempting to give a simple physical picture of the quasiparticle-pair current as being due to a cutoff frequency in the junction. ${ }^{2}$ It has been shown theoretically ${ }^{10,11}$ and experimentally ${ }^{12-14}$ that the ac Josephson current has a singularity at the energy gap $2 \Delta$ and decays logarithmically above it (the Riedel peak). If we set $\omega_{c}=2 \Delta / \hbar$ and $\omega_{1}=k I_{1} R=2 \Delta / \hbar$, we find from Eq. (4) that

$$
\sigma_{1} / \sigma_{0}=-\left[1+(\hbar \omega / 2 \Delta)^{2}\right]^{-1}
$$

Using $f=10 \mathrm{GHz}$ and $2 \Delta / h=500 \mathrm{GHz}$ as in Ref. 8, we find $\sigma_{1} / \sigma_{0} \approx-0.99$, which compares well to the reported value $-0.9 \pm 0.1$. In the limit $\Delta \rightarrow 0\left(T \rightarrow T_{c}\right)$, we find $\sigma_{1} / \sigma_{0} \rightarrow 0$ as theory predicts. ${ }^{9}$

The analog can be improved in several ways. A nonlinear $R$ could be used to simulate the changes in the quasiparticle resistance at the energy gap. A shunt capacitor outside the loop [i.e., an extra term $j \omega C$ in $\mathrm{Eq}$. (4)] would give rise to the plasma resonance.$^{8,15}$ A shunt resistor outside the loop would give an Ohmic current path as observed in low-resistance point contacts. Finally, at least in principle, a low-pass filter which gives the right shape of the Riedel peak and the decay above the energy gap might be used. This in turn would change the $\cos \phi$ conductivity of Eq. (4) slightly.

We have built two of these analog $s^{16}$ with the following characteristics: (a) $f_{0}=5 \mathrm{kHz}, f_{\mathrm{c}}=100 \mathrm{~Hz}$, and $k=100$ $\mathrm{Hz} / \mathrm{V}$; (b) $f_{0}=65 \mathrm{MHz}, f_{c}=100 \mathrm{kHz}$, and $k=40 \mathrm{kHz} / \mathrm{V}$. For the high-frequency one we can display the $I-V$ curve on an oscilloscope. The rf-induced steps show the regular Bessel function behavior with applied power, and we have been able to see the plasma resonance ${ }^{8,15}$ by including a capacitor.

A new scheme for a Josephson junction analog has been presented, and it is shown that the appearance of the $\cos \phi$ conductivity is a consequence of having a highfrequency rolloff in the ac Josephson current. We are thus led to believe that, because of high-frequency cutoff in the supercurrent, a quasiparticle-pair conductivity may well occur also in point contacts and weak links.

The authors wish to thank Professor Dr. K. Særmark, Professor Dr. Sidney Shapiro, Dr. J. Mygind, and U. K. Poulsen for numerous discussions that contributed greatly to this work.

${ }^{1}$ B.D. Josephson, Phys. Lett. 1, 251 (1962).

${ }^{2}$ B. D. Josephson, Rev. Mod. Phys. 36, 216 (1964).

${ }^{3}$ B.D. Josephson, Advan. Phys. 14, 419 (1965).

${ }^{4}$ N. R. Werthamer and Sidney Shapiro, Phys. Rev. 164, 523

(1967).

${ }^{5}$ C. A. Hamilton, Rev. Sci. Instr. 43, 445 (1972).

${ }^{6}$ D. B. Sullivan and J.E. Zimmerman, Am. J. Phys. 39, 1505 (1971).

${ }^{7}$ H. Nyquist, Bell Syst. Tech. J. 11, 126 (1932).

${ }^{8}$ N. F. Pedersen, T. F. Finnegan, and D. N. Langenberg,

Phys. Rev. B 6, 4151 (1972).

${ }^{9}$ U. K. Poulsen, Phys. Lett. A 41, 195 (1972).

${ }^{10} \mathrm{E}$. Riedel, Z. Naturforsch. A 19, 1634 (1964).

${ }^{11}$ N. R. Werthamer, Phys. Rev. 147, 255 (1966).

${ }^{12}$ C. A. Hamilton and Sidney Shapiro, Phys. Rev. Lett. 26, 426 (1971).

${ }^{13}$ S.A. Buckner, T. F. Finnegan, and D.N. Langenberg, Phys. Rev. Lett. 28, 150 (1972).

${ }^{14}$ C. A. Hamilton, Phys. Rev, B 5, 912 (1972).

${ }^{15}$ A.J. Dahm, A. Denenstein, T. F. Finnegan, D. N. Langen-

berg, and D.J. Scalapino, Phys. Rev. Lett. 20, 859 (1968).

${ }^{16}$ Details will be presented elsewhere. 